\title{
Applications of kidney organoids derived from human pluripotent stem cells
}

\author{
Yong Kyun $\mathrm{Kim}^{1,2}$, Sun Ah Nam ${ }^{1,2}$, and Chul Woo Yang ${ }^{2,3}$
}

\begin{abstract}
${ }^{1}$ Cell Death Disease Research Center, ${ }^{2}$ Department of Internal Medicine, ${ }^{3}$ Convergent Research Consortium for Immunologic Disease, and Division of Nephrology, College of Medicine, The Catholic University of Korea, Seoul, Korea
\end{abstract}

Received: June 11, 2018

Accepted: June 18, 2018

\section{Correspondence to}

Chul Woo Yang, M.D.

Convergent Research Consor-

tium for Immunologic Disease and Department of Internal

Medicine, College of Medicine,

Seoul St. Mary's Hospital, The

Catholic University of Korea, 222

Banpo-daero, Seocho-gu, Seoul

06591, Korea

Tel: +82-2-2258-6037

Fax: +82-2-22258-6917

E-mail:yangch@catholic.ac.kr

This paper was contributed by Korean Society of Nephrology.
The establishment of protocols to differentiate kidney organoids from human pluripotent stem cells provides potential applications of kidney organoids in regenerative medicine. Modeling of renal diseases, drug screening, nephrotoxicity testing of compounds, and regenerative therapy are attractive applications. Although much progress still remains to be made in the development of kidney organoids, recent advances in clustered regularly interspaced short palindromic repeat (CRISPR)-CRISPR-associated system 9 (Cas9) genome editing and three-dimensional bioprinting technologies have contributed to the application of kidney organoids in clinical fields. In this section, we review recent advances in the applications of kidney organoids to kidney disease modelling, drug screening, nephrotoxicity testing, and regenerative therapy.

Keywords: Bioprinting; Clustered regularly interspaced short palindromic repeats; Kidney; Organoids; Transplantation

\section{INTRODUCTION}

Kidney organoids are generated from human pluripotent stem cells (hPSCs) in vitro [1-4]. Kidney organoids derived from hPSCs contain nephrogenic progenitor cells of multiple lineages that can recapitulate kidney development [5]. A direct comparison of gene expression and localization between kidney organoids in vitro and human kidneys revealed that podocytes derived from hPSCs resemble podocytes in vivo at the capillary loop stage (CLS) of glomerular development [5]. Several review articles describing differentiation of kidney organoids from hPSCs have been published. Here, we focus on the applications of kidney organoids derived from hPSCs.

\section{APPLICATIONS OF KIDNEY ORGANOIDS DE- RIVED FROM HUMAN hPSCs}

Kidney organoids derived from hPSCs have potential applications in regenerative medicine, as well as in the modeling of renal diseases, drug screening, and nephrotoxicity testing of compounds. Although more research is needed to improve differentiation protocols and obtain fully functional kidney organoids, clustered regularly interspaced short palindromic repeat (CRIS- 


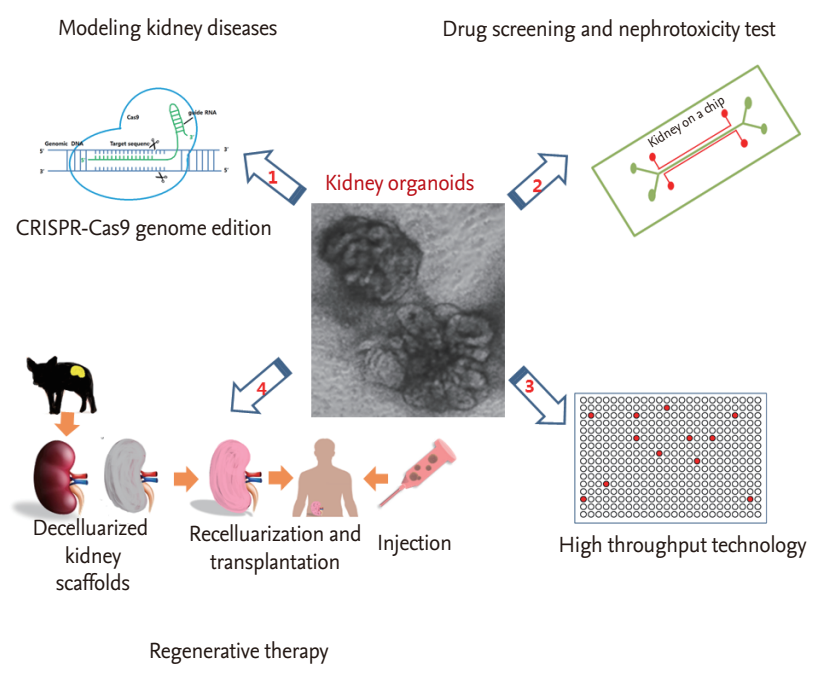

Figure 1. Schematic presentation of the clinical application of kidney organoids derived from human pluripotent stem cells. CRISPR, clustered regularly interspaced short palindromic repeat.

PR)-CRISPR-associated system 9 (Cas9) genome editing and three-dimensional $(3 \mathrm{D})$ bioprinting have facilitated realization of functional kidney organoids and their applications in clinical fields. In this section, we review recent advances in the applications of kidney organoids in kidney disease modelling, drug screening, nephrotoxicity testing, and regenerative therapy (Fig. 1).

\section{DISEASE MODELING}

Kidney disease modeling using kidney organoids derived from hPSCs has revealed novel mechanisms of kidney diseases and allows drug screening to develop new therapies. Advanced technologies for differentiating kidney organoids from hPSCs, as well as efficient genome editing systems, such as the CRISPR-Cas9 system, have enabled researchers to model human kidney diseases.

One of the most important issues in this field is the modeling of renal diseases with a genetic basis. Autosomal dominant polycystic kidney disease (ADPKD) is the most common genetic disease, and is present in approximately $5 \%$ of patients with total end-stage renal disease (ESRD). Numerous fluid-filled cysts form and grow in both kidneys, accompanied by interstitial fibro- sis, resulting in chronic kidney disease (CKD) in $50 \%$ of patients by the age of 60 years [6]. ADPKD is associated with mutations in the $P K D 1$ and $P K D 2$ genes [6], which encode polycystin-1 and polycystin-2, respectively $[6,7]$. The mechanism for the development of ADPKD remains unclear and treatment options for ADPKD are limited.

Freedman's research group modeled PKD using kidney organoids derived from hPSCs $[8,9]$. They produced hPSCs with loss-of-function mutations in either PKD1 or PKD2 using CRISPR-Cas9 genome editing and differentiated these mutant hPSCs into kidney organoids. CRISPR-mutant PKD1 and PKD2 knockout kidney organoids (PKD organoids) had cysts in the kidney tubules, phenocopying ADPKD.

PKD organoids derived from hPSCs have major advantages over currently available kidney disease models, such as PKD1 or PKD2 mutant mouse or renal cells from patients with ADPKD [8-11]. Although PKD1 and PKD2 mouse mutants have provided valuable insight into disease mechanisms, the survival time of $P K D 1$ and $P K D 2$ knockout mice is very short, and $P K D 1$ and $P K D 2$ heterozygotes have only very mild cystic disease, unlike human ADPKD [12]. Therefore, PKD organoids derived from hPSCs may be a better model of human ADPKD. In addition, PKD organoids are more accessible than animal models and their use does not require ethical approval. Cultures of renal cells from patients with ADP$\mathrm{KD}$ can also be used to model ADPKD in vitro [13]. However, this system suffers from heterogeneities in the cell sources and the epigenetic background of each patient; thus, the results need to be interpreted with caution $[11,13]$. In contrast, CRISPR-mutant PKD organoids derived from hPSCs have isogenic negative controls, which allows causality to be more clearly established when investigating disease mechanisms $[8,9,14]$.

PKD organoids can not only be used to recapitulate the phenotypic characteristics of ADPKD, but also to investigate the mechanisms of ADPKD cystogenesis [8]. Freedman and colleagues [8] established a highly efficient culture system for PKD organoids to model PKD cystogenesis. They cultured PKD organoids in low-attachment plates instead of in the previous adherent culture system, which resulted in the formation of larger cysts phenotypically resembling PKD [8]. In this culture system, the cystogenesis rate of the PKD mutant organ- 
oids was much higher than that of isogenic control organoids. Using this system, the role of the extracellular matrix (ECM) microenvironment in the cystogenesis of PKD was investigated [8]. The authors provided direct evidence for the role of the ECM microenvironment in cystogenesis in $\mathrm{PKD}$, by embedding the PKD organoids into collagen droplets. Embedding the PKD1 mutant organoids into collagen droplets dramatically decreases cyst formation, but removing the adherent cues abolished the ability of collagen droplets to reduce cyst size [8]. The authors also reported that the size of organoids increased, and cystogenesis was promoted, when cyclic adenosine monophosphate (cAMP) was added [8]. These approaches provide valuable insight into the underlying mechanisms of ADPKD and could aid in the development of therapeutics.

Mutations in vital genes in podocytes may cause podocytopathies, such as focal segmental glomerulosclerosis (FSGS), which is one of the leading glomerular-related causes of ESRD and has diverse genetic origins $[15,16]$. Podocalyxin (PODXL) is highly localized on the apical and lateral surfaces of podocytes and may be a candidate gene for FSGS [17]. Kim et al. [5] demonstrated the functional role of PODXL in the development of podocyte disease using kidney organoids. They generated gene-edited PODXL ${ }^{-/-}$hPSCs and isogenic controls using the CRISPR-Cas9 system and differentiated these hPCSs to form kidney organoids. PODXL ${ }^{-/-} \mathrm{hP}$ SC-podocytes had defects in basal junctional migration. PODXL ${ }^{-1-}$ hPSC-podocytes also failed to assemble microvilli, with the absence of microvilli being accompanied by a reduction in lateral spaces between adjacent podocytes [5]. Importantly, these findings in PODXL-/hPSC-podocytes were validated in the CLS glomeruli of PODXL-deficient mice [5]. Furthermore, human patients with biallelic loss-of-function mutations in PODXL may have congenital nephrotic syndrome [17]. That study demonstrated the potential usefulness of kidney organoids derived from hPSCs to model podocyte diseases.

There are at least 150 different genetic renal disorders worldwide and nearly all children with a genetic renal disorder progress to ESRD [18]. Kidney organoid systems combined with CRISPR genome editing can aid in the modeling of genetic renal disorders, which is likely to provide valuable insight facilitating the development of new therapies.

\section{DRUG SCREENING AND NEPHROTOXICITY TESTING}

\section{Renal tubular cell-based approaches}

Most newly developed drugs have nephrotoxic potential, and predicting nephrotoxicity during preclinical drug development is essential [19]. Several cell types have been used for in vitro nephrotoxicity assays, and each cell type has advantages and disadvantages (Table 1) $[19,20]$. Madin-Darby canine kidney cells, which are a well-established cell line, form a polarized tight monolayer, but show differences from those in humans and are poor predictors of nephrotoxicity [2o].

Proximal tubular (PT) cells have numerous active transporters to reabsorb filtered drug metabolites, resulting in a high intracellular accumulation of drug metabolites [19-22]. Thus, nephrotoxic drugs are likely to affect PT cells [20]. For this reason, PT cells have been widely used to develop in vitro platforms for drug screening or nephrotoxicity testing [20]. Proximal-like porcine kidney 1 (LLC-PK1) cells (porcine PT cell line), human kidney 2 (HK-2) cells (immortalized human PT cell line), and primary renal PT epithelial cells from different donors are widely used as cell sources for PT cells. In vitro platforms using PT cells have been reported; their sensitivity and accuracy are within the range of $70 \%$ to $90 \%$ depending on the PT cell type $[19,23,24]$. However, HK-2 cells have few transporters or proximal tubule characteristics $[25,26]$ and show a tendency to lose their transepithelial transport function once they undergo the epithelial-to-mesenchymal transition [27]. Primary human renal PT epithelial cells have transepithelial transporters and express metabolic enzymes, and are also available for a broad range of biomarker assays. This cell line is suitable for nephrotoxicity testing and show high predictivity $[20,28,29]$. However, these cells rapidly lose their PT characteristics during prolonged culture, and there is donor-to-donor variability and possible contamination with other renal cell types when the cells are isolated from donors due to heterogeneity of the kidney [20,30].

\section{hPSC-based approaches}

Differentiating hPSCs into PT epithelial cell-like cells is an alternative and attractive approach to establish an in vitro model. One research group reported an accuracy 


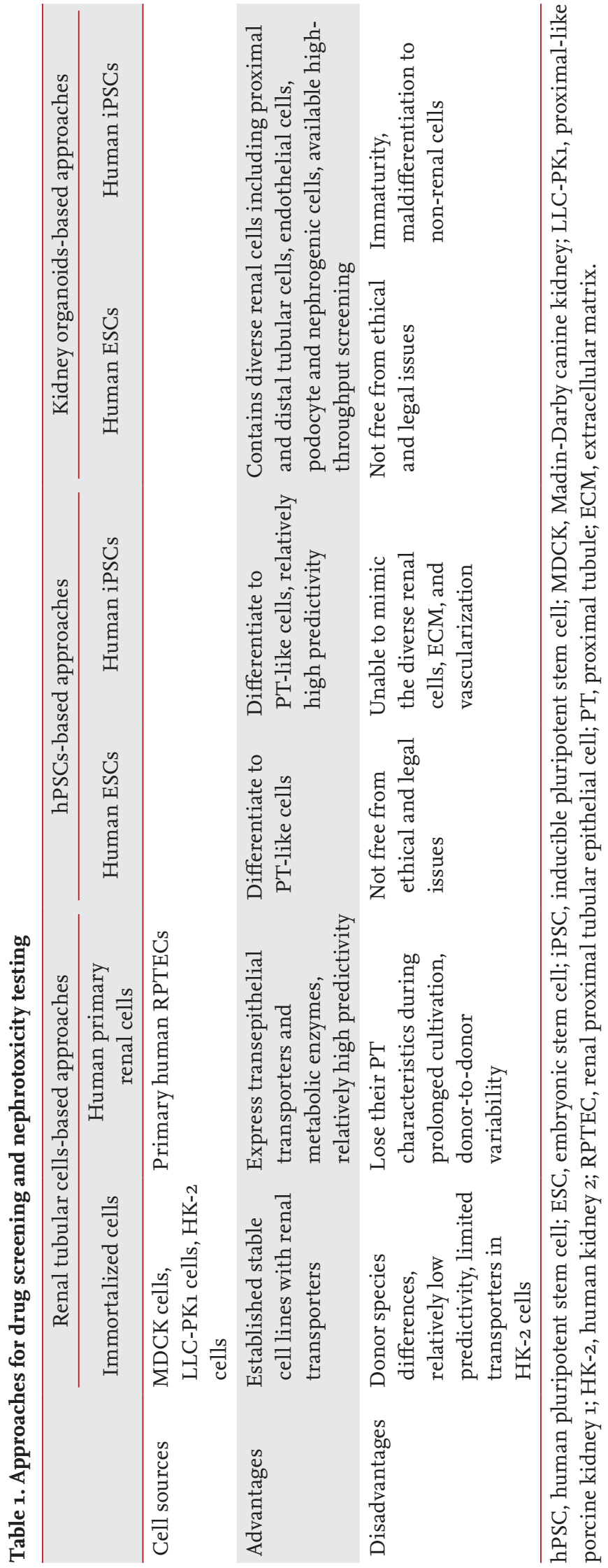

of $76 \%$ for an in vitro model based on embryonic stem cell-derived human renal PT-like cells [31]. However, embryonic stem cell-derived human renal PT-like cells have not been sufficiently characterized [32] and their use is not free from ethical and legal issues.

Thus, human inducible pluripotent stem cell (iP$\mathrm{SC}$ )-based models are alternative and attractive in vitro models. A protocol to differentiate human iPSCs into PT-like cells and establish a human iPSC-based model for predicting nephrotoxicity has been published [33]. Using this protocol, PT-like cells with a purity $>90 \%$ were obtained from iPSCs. This human iPSC-based model was used to predict the toxicity of 30 compounds to PT cells; the training accuracy of the model was $99.8 \%$, and the test accuracy was $87.0 \%$ [33]. Although the model can predict nephrotoxicity with reasonable accurately, it does not mimic the diversity of cells, ECM, or vascularization associated with actual human kidneys. Thus, a human iPSC-based model is required to extrapolate in vitro evidence to the in vivo environment [20].

\section{Kidney organoids derived using hPSC-based ap- proaches}

Cell-to-cell and cell-to-ECM interactions control cell phenotypes and functions in vivo [34-36]. Maintaining these interactions is required to maintain tissue-like responses in an in vitro model in the context of drug efficacy and toxicity screening [37]. Two-dimensional cultures on culture plastic using a single renal cell type may have difficulty in recapitulating these complex conditions. In contrast to conventional kidney cell lines or iPSC-derived PT cells, individual kidney organoids have all of the major components of developing proximal nephrons, namely tubular cells, endothelial cells, nephron progenitors, and podocyte-like cells $[1,4,9]$, and are therefore promising systems for drug screening and nephrotoxicity testing.

Some research groups have tested the nephrotoxicity of gentamycin or cisplatin on kidney organoids derived from hPSCs $[1,4,9]$. After kidney organoids were treated with gentamycin or cisplatin, increased expression of kidney injury molecule-1, a clinical biomarker of acute kidney injury (AKI) $[1,9]$, or cleaved caspase 3, a marker of apoptosis [4], was observed by immunofluorescence staining or real-time quantitative polymerase chain reaction. These data support the use of kidney organoids 
as a platform for nephrotoxicity testing. However, more nephrotoxic drugs and non-nephrotoxic compounds have to be evaluated before kidney organoids can be used as a drug testing platform, and the results must be compared with tubular cell-based models or hPSC-based models.

\section{HIGH-THROUGHPUT APPLICATIONS}

Accurate high-throughput methods to screen large numbers of compounds are essential to reduce time and cost during drug development [38]. Some groups have developed a predictive screening platform with high efficiency and accuracy by combining cell-based (HK-2 cells and human primary renal PT cells) and computational methods $[38,39]$.

Kidney organoids derived from hPSCs have great potential in high-throughput screening because of their specificity to humans and ability to model complex kidney disease phenotypes [40]. However, the complexity of kidney organoid cultures has been a significant obstacle to miniaturization and automation [40]. Czerniecki et al. [40] established a fully automated, high-throughput screening-compatible system using liquid-handling robots and a 21-day organoid culture protocol, from plating to analysis via differentiation. They demonstrated that this high-throughput screening system improved differentiation of kidney organoids. Using this system, they modeled nephrotoxicity and disease phenotypes and quantified the system in terms of its safety and prediction efficacy. They also applied the system to drug screening. They cultured PKD mutant organoids in 96-well plates treated with different compounds and demonstrated that blebbistatin, a specific inhibitor of non-muscle myosin II, induces a significant increase in cyst formation, which revealed an unexpected role for myosin in polycystic kidney disease [40].

Although limitations, for example in terms of the immaturity or functionality of kidney organoids derived from hPSCs still remain, high-throughput systems based on kidney organoids derived from hPSCs may be a valuable tool for new drug development or nephrotoxicity testing.

\section{APPLICATIONS WITH 3 D BIOPRINTING}

Another important application of kidney organoids is 3D bioprinting. Three-dimensional bioprinting enables layer-by-layer precise positioning of biological and biochemical materials, and living cells, with spatial control of the placement of functional components to fabricate complex 3D living tissues or organs [41]. Three-dimensional bioprinting has advantages over non-biological printing because $3 \mathrm{D}$ bioprinting can be used to fabricate complex structures, by choosing different materials, cell types, and growth and differentiation factors. Three-dimensional bioprinting provide opportunities to meet technical challenges related to the sensitivity of living cells and the tissue construction [41]. Thus, application of ${ }_{3} \mathrm{D}$ bioprinting technologies to regenerative medicine is an important issue.

Three-dimensional bioprinting can be applied in two ways to regenerative medicine for kidney diseases. Drug screening using a drug library is important during new drug development, and nephrotoxicity testing should be performed before clinical use of a newly developed compound. For drug screening or nephrotoxicity testing, $3 \mathrm{D}$ bioprinting can generate biologically mini kidney tissues, the so-called "kidney on a chip."

One research group has developed a bioprinting method for printing $3 \mathrm{D}$ convoluted renal proximal tubules embedded in ECM in vitro on customized perfusion chips with a microfluidic network, by precisely co-printing bioink with multiple materials [42]. Using this system, kidney tissue-like epithelium with phenotypic and functional properties similar to those of real kidney tissue can be obtained. For this chip to be used in drug screening applications, the presence of appropriate vasculature may be essential to reproduce organ-scale drug delivery pharmacokinetics, because vasculature is important when mimicking organ-level functions in micro-tissues [43]. Perfusable 3D proximal tubules promote the formation of a tissue-like epithelium with improved phenotypic and functional properties and mimic blood flow in vascular channels. Although no nephrogenic progenitor cells are used in this system, it could facilitate drug screening and mechanistic drug studies.

Organovo Inc. (San Diego, CA, USA) recently generated $3 \mathrm{D}$ bioprinted kidney tissue for compound screening and disease modelling [44]. They optimized a method to 
form a polarized layer of renal epithelium on top of the interstitial layer, and a basement membrane between the layers; this tissue may provide insight into the effects of cell-cell interactions on the nephrotoxicity of a compound [44].

Because kidney organoids are composed of diverse renal cells, including podocytes, tubular cells, endothelial cells, and stromal cells, and form nephron-like structures with functionality, they are suitable biomaterials for $3 \mathrm{D}$ bioprinting to produce efficient kidneys on a chip. Several research groups are trying to develop new methods to realize kidney tissues on a chip using kidney organoids derived from hPSCs.

Another application of $3 \mathrm{D}$ bioprinting of kidney organoids is generating organotypic scaffolds for transplantable bioengineered kidneys. One research group generated bioengineered bladders by $3 \mathrm{D}$ bioprinting and transplanted them into seven patients with non-functional bladders [45]. They generated a biodegradable scaffold and seeded urothelial and muscle cells into the bioengineered bladder scaffolds [45]. However, unlike the bladder, vessels, or trachea bioengineered by 3D bioprinting [46], the kidney contains 1 million nephrons consisting of diverse cells with different functions and origins. Given the complex structure and functional nature of the kidney, and the absence of an organized urinary excretion system in kidney organoids, bioprinted functional kidney tissues have not been realized despite advances in bioprinting technologies [47].

\section{REGENERATIVE THERAPY USING KIDNEY OR- GANOIDS}

The incidence and prevalence of CKD is increasing in developed countries, leading to ESRD requiring renal replacement therapy, such as dialysis or kidney transplantation [48]. However, dialysis is limited by high mortality, and kidney transplantation is limited by the shortage of donor organs [49-53]. Therefore, new therapeutic options are urgently needed. Regenerative medicine, in which the nephrons lost during the progression of CKD are replaced by stem cells, is a potentially attractive therapeutic option for CKD. In this section, we review the possible regenerative medicine options afforded by kidney organoids.

\section{Transplantation and cell therapy using kidney or- ganoids}

Kidney organoids have multi-lineage nephrogenic progenitor cells, and immunosuppression is not needed to transplant kidney organoids derived from patients. Thus, transplanting kidney organoids is an attractive regenerative therapy. However, this may not be achievable in a short time frame, for several reasons. The evidence of functionality of kidney organoids remains insufficient. It is not easy to generate the urine collecting system and connect it with the host urinary system. The safety of iPSCs and the immune barrier in ESCs also limit transplantation of kidney organoids derived from hPSCs [10].

Nevertheless, some researchers have reported encouraging data [2,54-57]. Taguchi et al. [2] generated metanephric nephron progenitor from mouse ESCs and transplanted them beneath the kidney capsule of immunodeficient mice. Tubulogenesis and formation of glomerular-like structures were observed in the transplanted graft. The transplanted glomeruli vascularized with the host circulation and contained red blood cells.

Another study also reported encouraging data. Sharmin et al. [55] generated human iPSC cell lines expressing green fluorescent protein in the NPHS locus using transcription activator-like effector nuclease-mediated homologous recombination, and then induced $3 \mathrm{D}$ primordial glomeruli from these iPSCs. They reported that the induced human podocytes derived from iPSCs exhibited apicobasal polarity, by showing the location of an in-basal domain with primary foot process-like structures that were connected with slit diaphragm-like structures. They developed a transplantation technique to effectively form glomeruli from human iPSC-derived nephron progenitors using spacers that released tension from the host kidney capsules. Using this technique, induced glomeruli were transplanted from human iPSCs into the mouse kidney subcapsular space, followed by treatment with vascular endothelial growth factor (VEGF) [55]. The transplanted human glomeruli were vascularized with the host mouse endothelial cells, and the iPSC-derived podocytes had numerous cell processes that accumulated around the fenestrated endothelial cells, forming slit diaphragm-like structures.

Another study reported functional vascularization with progressive morphogenesis of human kidney or- 
HNA staining

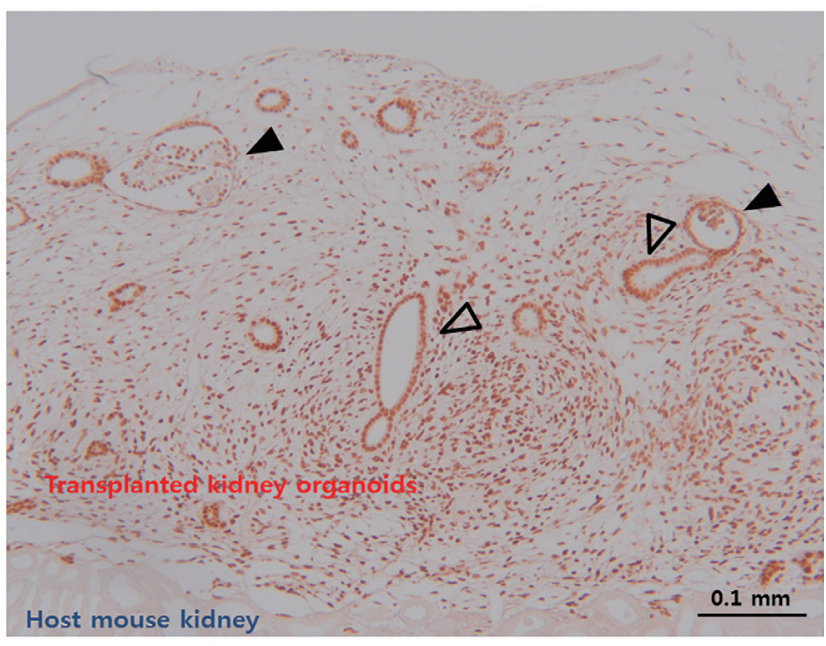

Figure 2. Representative images immunohistochemical staining images (with human nuclear antibody [HNA]) after transplantation of kidney organoids derived from human pluripotent stem cells (hPSCs) into kidney of nonobese diabetic/severe combined immunodeficiency (NOD-SCID) mouse. Scale bars, $0.1 \mathrm{~mm}$. Transplanted kidney organoids derived from hPSCs grew with nephron-like structures and the cells in transplanted kidney organoids predominantly originated from the human inducible pluripotent stem cells. Black arrowheads indicate the glomerulus-like structure and white arrowheads indicate the tubule-like structure in transplanted graft.

ganoids after renal subscapular transplantation. van den Berg et al. [57] transplanted kidney organoids derived from hPSCs into mouse kidney subcapsular spaces and reported that the transplanted hPSC-derived kidney organoids develop host-derived vascularization in the absence of any exogenous VEGF. They also showed functional glomerular perfusion with connection to host mouse vascular networks by in vivo imaging of the transplanted kidney organoids under the mouse kidney capsule. They revealed that transplantation induced the formation of a slit diaphragm structure consisting of the glomerular basement membrane, fenestrated endothelial cells, and podocyte foot processes in an ultrastructural analysis. They also observed specialized (i.e., more polarized and segmented) tubular epithelium in transplanted kidney organoids compared with non-transplanted organoids. Our laboratory also transplanted kidney organoids derived from hPSCs beneath the renal capsular space of nonobese diabetic/severe combined immunodeficiency (NOD-SCID) mice. The transplanted kidney organoids vascularized with the host mouse and developed nephron-like structures (Fig. 2).

The therapeutic effectiveness of transplanting kidney organoids for kidney diseases has not yet been described but can be predicted based on previous reports $[54,56]$. Toyohara et al. [56] induced hPSCs into $\mathrm{OSR}_{1}{ }^{+} \mathrm{SIX}_{2}{ }^{+}$renal progenitors, which were capable of reconstituting ${ }_{3} \mathrm{D}$ proximal renal tubule-like structures in vitro and in vivo. They transplanted these human iPSC-derived renal progenitors cells into the kidney subcapsular space of mice in an ischemia/reperfusion injury AKI model, which resulted in suppression of the elevation of blood urea nitrogen and serum creatinine levels and attenuation of histopathological changes, such as tubular necrosis, tubule dilatation with casts, and interstitial fibrosis. Their data indicate that the renoprotective benefits originated from the trophic effects produced by the transplanted renal progenitor cells.

Another study was performed using a cisplatin injury-induced AKI mouse model [54]. Imberti et al. [54] induced renal progenitors from human iPSCs and infused them intravenously into mice with cisplatin-induced AKI. Injecting human iPSCs derived from renal progenitor cells restored renal function and structure in mice with cisplatin-induced AKI. Interestingly, different from Toyohara et al. [56], these authors reported robust engraftment of human iPSCs derived from renal progenitor cells into damaged tubules of the host mouse kidney. Although it remains unclear whether renal recovery from AKI is due to the integrated human iPSCs derived from renal progenitor cells, or to regenerated tubular cells via paracrine effects arising from infused iPSC-derived renal progenitor cells, these data provide the basis for future applications of kidney organoids derived from hPSCs in regenerative therapy.

\section{Recellularization of decellularized kidney scaffolds}

Transplantation of a regenerated kidney using kidney scaffolds may be an attractive therapeutic option for patients with ESRD. Recellularization of decellularized kidney scaffolds with renal progenitor cells and endothelial cells is important for generating bioengineered kidneys for clinical use in the future, considering the successful clinical transplantation of recellularized tracheal scaffolds from decellularized cadaveric trachea [58,59].

One group performed experimental orthotopic trans- 
plantation of re-populating decellularized kidneys [6o]. They seeded decellularized rat kidney scaffolds with rat neonatal kidney cells via the ureter and human umbilical vascular endothelial cells via the renal artery, and then perfused it in a whole-organ bioreactor. The decellularized rat kidney scaffolds were successfully recellularized. The repopulated kidneys produced rudimentary urine in vitro. Furthermore, when these bioengineered kidneys were transplanted in an orthotopic position in rats, the grafts were perfused by the recipient's circulation and produced urine through the ureteral conduit in vivo [6o].

Achieving an appropriate combination of cell types is one of the main issues in recellularization of decellularized kidney scaffolds. Thus, various combinations of cell types, such as renal cells with endothelial cells, including mouse ESCs [61,62], human iPSC-derived endothelial cells with human renal cortical tubular epithelial cells [63], rat aorta endothelial cells with rat epithelial tubular cells [64], and human umbilical vascular endothelial cells with rat neonatal kidney cells [6o] have been explored.

Recellularization with renal progenitor cells and endothelial cells derived from human iPSCs results in abundant repopulation of decellularized kidney scaffolds and formation of a vascular network [65]. Du et al. [65] generated $\mathrm{PAX}^{+}$renal progenitor cells and endothelial cells from human iPSCs, which repopulated decellularized kidney scaffolds. Interestingly, the expression levels of genes related to renal development were higher in the recellularized group that had $\mathrm{PAX}^{+}$renal progenitor cells and endothelial cells than in the group that only had $\mathrm{PAX}^{+}$renal progenitor cells. When the recellularized kidney scaffolds were implanted in immunodeficient mice, glomerular assembly and function were achieved by recellularization with renal progenitor cells and endothelial cells. That study provided evidence for the vital role of endothelial cells in the generation of a functional whole kidney from a kidney scaffold, and highlighted the usefulness of iPSC-derived nephrogenic progenitors.

Numerous obstacles must be overcome to clinically apply bioengineered kidneys: optimization of cell-seeding protocols to create human-sized scaffolds, upscaling of biomimetic organ cultures, and differentiation and expansion of the required cell types from clinically fea- sible sources [6o]. Pigs may be suitable for decellularized kidney scaffolds because of the similarity in size and microarchitecture between pig and human kidneys [66]. To achieve repopulation with a broader variety of different kidney-like cell types and appropriate organization, kidney organoids derived from hPSCs may be suitable cell sources for recellularization. However, whether kidney organoids derived from iPSCs can be used as cell sources to seed decellularized kidney scaffolds has not been evaluated.

\section{OBSTACLES}

Despite biotechnological advances, applications of kidney organoids derived from human iPSCs have just begun and there are many obstacles to overcome for their clinical use. The safety of kidney organoids is an important concern for clinical use. Human iPSCs can form tumors. Some populations of progenitor cells in kidney organoids derived from iPSCs are not fully differentiated. Thus, regenerative therapy using kidney organoids may not be free from the risk of tumor development. Fortunately, no study thus far has reported the development of tumors in patients treated with hPSCs [67]. Quality control of iPSCs to ensure that they have a normal karyotype is also required [67].

Five different protocols have been published to differentiate kidney organoids from hPSCs [1-4]. The phenotypic characteristics of kidney organoids vary according to the differentiation protocol $[47,68]$. No protocol generates kidney organoids that completely recapitulate the complex structural and functional nature of kidneys, which limits their efficacy for kidney disease modeling and regenerative therapies. More precise and standardized differentiation protocols are needed.

\section{CONCLUSIONS}

Many people suffer from CKD [49,50] and regenerative medicine may be a promising therapeutic option. Recent advances in differentiating kidney organoids from hPSCs and biotechnological advances have allowed for diverse applications of kidney organoids in the clinical setting. Precise modeling of genetic kidney diseases by 
CRISPR genome editing and advanced high-throughput technologies is a powerful tool for drug screening and developing new therapies. Further development of the "kidney on a chip," based on kidney organoids and 3D-bioprinting techniques, will hopefully allow for nephrotoxicity evaluations with accuracy similar to that in vivo. Regenerative therapy using kidney organoids might be a game changer in the battle against kidney diseases. Despite the challenges that need to be overcome for clinical application of kidney organoids, kidney organoids derived from hPSCs appear to have great potential.

\section{Conflict of interest}

No potential conflict of interest relevant to this article was reported.

\section{Acknowledgments}

This research was supported by the Basic Science Research Program through the National Research Foundation of Korea (NRF), funded by the Ministry of Education, Science, and Technology (NRF-2018R1A2B6003440).

\section{REFERENCES}

1. Morizane R, Lam AQ, Freedman BS, Kishi S, Valerius MT, Bonventre JV. Nephron organoids derived from human pluripotent stem cells model kidney development and injury. Nat Biotechnol 2015;33:1193-1200.

2. Taguchi A, Kaku Y, Ohmori T, et al. Redefining the in vivo origin of metanephric nephron progenitors enables generation of complex kidney structures from pluripotent stem cells. Cell Stem Cell 2014;14:53-67.

3. Takasato M, Er PX, Becroft M, et al. Directing human embryonic stem cell differentiation towards a renal lineage generates a self-organizing kidney. Nat Cell Biol 2014;16:118-126.

4. Takasato M, Er PX, Chiu HS, et al. Kidney organoids from human iPS cells contain multiple lineages and model human nephrogenesis. Nature 2015;526:564-568.

5. Kim YK, Refaeli I, Brooks CR, et al. Gene-edited human kidney organoids reveal mechanisms of disease in podocyte development. Stem Cells 2017;35:2366-2378.

6. Peters DJ, Breuning MH. Autosomal dominant polycystic kidney disease: modification of disease progression. Lan- cet 2001;358:1439-1444.

7. Willey CJ, Blais JD, Hall AK, Krasa HB, Makin AJ, Czerwiec FS. Prevalence of autosomal dominant polycystic kidney disease in the European Union. Nephrol Dial Transplant 2017;32:1356-1363.

8. Cruz NM, Song X, Czerniecki SM, et al. Organoid cystogenesis reveals a critical role of microenvironment in human polycystic kidney disease. Nat Mater 2017;16:11121119.

9. Freedman BS, Brooks CR, Lam AQ, et al. Modelling kidney disease with CRISPR-mutant kidney organoids derived from human pluripotent epiblast spheroids. Nat Commun 2015;6:8715.

10. Freedman BS. Modeling kidney disease with iPS cells. Biomark Insights 2015;10(Suppl 1):153-169.

11. Romagnani P. Organoids: modelling polycystic kidney disease. Nat Mater 2017;16:1058-1059.

12. Happe H, Peters DJ. Translational research in ADPKD: lessons from animal models. Nat Rev Nephrol 2014;10:587601.

13. Carone FA, Nakamura S, Bacallao R, Nelson WJ, Khokha M, Kanwar YS. Impaired tubulogenesis of cyst-derived cells from autosomal dominant polycystic kidneys. Kidney Int 1995;47:861-868.

14. Cruz NM, Freedman BS. CRISPR gene editing in the kidney. Am J Kidney Dis 2018;71:874-883.

15. De Vriese AS, Sethi S, Nath KA, Glassock RJ, Fervenza FC. Differentiating primary, genetic, and secondary FSGS in adults: a clinicopathologic approach. J Am Soc Nephrol 2018;29:759-774.

16. Devarajan P, Spitzer A. Towards a biological characterization of focal segmental glomerulosclerosis. Am J Kidney Dis 2002;39:625-636.

17. Kang HG, Lee M, Lee KB, et al. Loss of podocalyxin causes a novel syndromic type of congenital nephrotic syndrome. Exp Mol Med 2017;49:e414.

18. Devuyst O, Knoers NV, Remuzzi G, Schaefer F; Board of the Working Group for Inherited Kidney Diseases of the European Renal Association and European Dialysis and Transplant Association. Rare inherited kidney diseases: challenges, opportunities, and perspectives. Lancet 2014;383:1844-1859.

19. Tiong HY, Huang P, Xiong S, Li Y, Vathsala A, Zink D. Drug-induced nephrotoxicity: clinical impact and preclinical in vitro models. Mol Pharm 2014;11:1933-1948.

20. Wilmer MJ, Ng CP, Lanz HL, Vulto P, Suter-Dick L, 
Masereeuw R. Kidney-on-a-chip technology for drug-induced nephrotoxicity screening. Trends Biotechnol 2016;34:156-170.

21. Anzai N, Jutabha P, Kanai Y, Endou H. Integrated physiology of proximal tubular organic anion transport. Curr Opin Nephrol Hypertens 2005;14:472-479.

22. Morrissey KM, Stocker SL, Wittwer MB, Xu L, Giacomini KM. Renal transporters in drug development. Annu Rev Pharmacol Toxicol 2013;53:503-529.

23. Li Y, Oo ZY, Chang SY, et al. An in vitro method for the prediction of renal proximal tubular toxicity in humans. Toxicol Res 2013;2:352-365.

24. Su R, Li Y, Zink D, Loo LH. Supervised prediction of drug-induced nephrotoxicity based on interleukin- 6 and -8 expression levels. BMC Bioinformatics 2014;15 Suppl 16:S16.

25. Jenkinson SE, Chung GW, van Loon E, Bakar NS, Dalzell AM, Brown CD. The limitations of renal epithelial cell line HK-2 as a model of drug transporter expression and function in the proximal tubule. Pflugers Arch 2012;464:601611.

26. Mutsaers HA, Wilmer MJ, van den Heuvel LP, Hoenderop JG, Masereeuw R. Basolateral transport of the uraemic toxin p-Cresyl sulfate: role for organic anion transporters? Nephrol Dial Transplant 2011;26:4149.

27. Slusser A, Bathula CS, Sens DA, et al. Cadherin expression, vectorial active transport, and metallothionein isoform 3 mediated EMT/MET responses in cultured primary and immortalized human proximal tubule cells. PLoS One 2015;10:e0120132.

28. Brown CD, Sayer R, Windass AS, et al. Characterisation of human tubular cell monolayers as a model of proximal tubular xenobiotic handling. Toxicol Appl Pharmacol 2008;233:428-438.

29. Lash LH, Putt DA, Cai H. Drug metabolism enzyme expression and activity in primary cultures of human proximal tubular cells. Toxicology 2008;244:56-65.

30. Van der Hauwaert C, Savary G, Gnemmi V, et al. Isolation and characterization of a primary proximal tubular epithelial cell model from human kidney by CD10/CD13 double labeling. PLoS One 2013;8:e66750.

31. Li Y, Kandasamy K, Chuah JK, et al. Identification of nephrotoxic compounds with embryonic stem-cellderived human renal proximal tubular-like cells. Mol Pharm 2014;11:1982-1990.

32. Narayanan K, Schumacher KM, Tasnim F, et al. Human embryonic stem cells differentiate into functional renal proximal tubular-like cells. Kidney Int 2013;83:593-603.

33. Kandasamy K, Chuah JK, Su R, et al. Prediction of drug-induced nephrotoxicity and injury mechanisms with human induced pluripotent stem cell-derived cells and machine learning methods. Sci Rep 2015;5:12337.

34. Cukierman E, Pankov R, Stevens DR, Yamada KM. Taking cell-matrix adhesions to the third dimension. Science 2001;294:1708-1712.

35. Cukierman E, Pankov R, Yamada KM. Cell interactions with three-dimensional matrices. Curr Opin Cell Biol 2002;14:633-639.

36. Geiger B, Bershadsky A, Pankov R, Yamada KM. Transmembrane crosstalk between the extracellular matrix: cytoskeleton crosstalk. Nat Rev Mol Cell Biol 2001;2:793805 .

37. Astashkina AI, Mann BK, Prestwich GD, Grainger DW. A 3-D organoid kidney culture model engineered for high-throughput nephrotoxicity assays. Biomaterials 2012;33:4700-4711.

38. Su R, Xiong S, Zink D, Loo LH. High-throughput imaging-based nephrotoxicity prediction for xenobiotics with diverse chemical structures. Arch Toxicol 2016;90:27932808.

39. Ramm S, Adler M, Vaidya VS. A high-throughput screening assay to identify kidney toxic compounds. Curr Protoc Toxicol 2016;69:9.

40. Czerniecki SM, Cruz NM, Harder JL, et al. High-throughput screening enhances kidney organoid differentiation from human pluripotent stem cells and enables automated multidimensional phenotyping. Cell Stem Cell 2018;22:929-940.

41. Murphy SV, Atala A. 3D bioprinting of tissues and organs. Nat Biotechnol 2014;32:773-785.

42. Homan KA, Kolesky DB, Skylar-Scott MA, et al. Bioprinting of $3 \mathrm{D}$ convoluted renal proximal tubules on perfusable chips. Sci Rep 2016;6:34845.

43. Osaki T, Sivathanu V, Kamm RD. Vascularized microfluidic organ-chips for drug screening, disease models and tissue engineering. Curr Opin Biotechnol 2018;52:116-123.

44. King S, Creasey O, Presnell S, Nguyen D. Design and characterization of a multicellular, three-dimensional (3D) tissue model of the human kidney proximal tubule FASEB J 2015;29(1 Suppl):LB426.

45. Atala A, Bauer SB, Soker S, Yoo JJ, Retik AB. Tissue-engineered autologous bladders for patients needing cysto- 
plasty. Lancet 2006;367:1241-1246.

46. Chang JW, Park SA, Park JK, et al. Tissue-engineered tracheal reconstruction using three-dimensionally printed artificial tracheal graft: preliminary report. Artif Organs 2014;38:E95-E105.

47. Chuah JKC, Zink D. Stem cell-derived kidney cells and organoids: recent breakthroughs and emerging applications. Biotechnol Adv 2017;35:150-167.

48. Jha V, Garcia-Garcia G, Iseki K, et al. Chronic kidney disease: global dimension and perspectives. Lancet 2013; 382:260-272.

49. Jin DC, Yun SR, Lee SW, et al. Current characteristics of dialysis therapy in Korea: 2016 registry data focusing on diabetic patients. Kidney Res Clin Pract 2018;37:20-29.

50. Jin DC, Yun SR, Lee SW, et al. Lessons from 30 years' data of Korean end-stage renal disease registry, 1985-2015. Kidney Res Clin Pract 2015;34:132-139.

51. Kiuchi MG, Mion D Jr. Chronic kidney disease and risk factors responsible for sudden cardiac death: a whiff of hope? Kidney Res Clin Pract 2016;35:3-9.

52. Jeong HY, Cho HJ, Kim SH, et al. Association of serum uric acid level with coronary artery stenosis severity in Korean end-stage renal disease patients. Kidney Res Clin Pract 2017;36:282-289.

53. Bae EH, Kim HY, Kang YU, Kim CS, Ma SK, Kim SW. Risk factors for in-hospital mortality in patients starting hemodialysis. Kidney Res Clin Pract 2015;34:154-159.

54. Imberti B, Tomasoni S, Ciampi O, et al. Renal progenitors derived from human iPSCs engraft and restore function in a mouse model of acute kidney injury. Sci Rep 2015:5:8826.

55. Sharmin S, Taguchi A, Kaku Y, et al. Human induced pluripotent stem cell-derived podocytes mature into vascularized glomeruli upon experimental transplantation. J Am Soc Nephrol 2016;27:1778-1791.

56. Toyohara T, Mae S, Sueta S, et al. Cell therapy using human induced pluripotent stem cell-derived renal progenitors ameliorates acute kidney injury in mice. Stem Cells Transl Med 2015;4:980-992.

57. van den Berg CW, Ritsma L, Avramut MC, et al. Renal subcapsular transplantation of PSC-derived kidney organoids induces neo-vasculogenesis and significant glomerular and tubular maturation in vivo. Stem Cell Reports 2018;10:751-765.

58. Gonfiotti A, Jaus MO, Barale D, et al. The first tissue-engineered airway transplantation: 5-year follow-up results. Lancet 2014;383:238-244.

59. Macchiarini P, Jungebluth P, Go T, Asnaghi MA, et al. Clinical transplantation of a tissue-engineered airway. Lancet 2008;372:2023-2030.

6o. Song JJ, Guyette JP, Gilpin SE, Gonzalez G, Vacanti JP, Ott HC. Regeneration and experimental orthotopic transplantation of a bioengineered kidney. Nat Med 2013;19:646-651.

61. Bonandrini B, Figliuzzi M, Papadimou E, et al. Recellularization of well-preserved acellular kidney scaffold using embryonic stem cells. Tissue Eng Part A 2014;20:14861498.

62. Guan Y, Liu S, Sun C, et al. The effective bioengineering method of implantation decellularized renal extracellular matrix scaffolds. Oncotarget 2015;6:36126-36138.

63. Caralt M, Uzarski JS, Iacob S, et al. Optimization and critical evaluation of decellularization strategies to develop renal extracellular matrix scaffolds as biological templates for organ engineering and transplantation. Am J Transplant 2015;15:64-75.

64. Hachisuka S, Sato Y, Yoshiike M, Nakazawa R, Sasaki H, Chikaraishi T. Enhanced recellularization of renal extracellular matrix scaffold under negative pressure. Integr Mol Med 2015;2:394-99.

65. Du C, Narayanan K, Leong MF, et al. Functional kidney bioengineering with pluripotent stem-cell-derived renal progenitor cells and decellularized kidney scaffolds. Adv Healthc Mater 2016;5:2080-2091.

66. Sampaio FJ, Pereira-Sampaio MA, Favorito LA. The pig kidney as an endourologic model: anatomic contribution. J Endourol 1998;12:45-50.

67. Wilm B, Tamburrini R, Orlando G, Murray P. Autologous cells for kidney bioengineering. Curr Transplant Rep 2016;3:207-220.

68. Morizane R, Bonventre JV. Kidney organoids: a translational journey. Trends Mol Med 2017;23:246-263. 